EPiC Series in Computing
Volume 70, 2020, Pages 226-237
$\begin{gathered}\text { Proceedings of the 12th International Conference } \\ \text { on Bioinformatics and Computational Biology }\end{gathered}$

\title{
Bioinformatics analysis of hereditary disease gene set to identify key modulators of myocardial remodeling during heart regeneration in zebrafish
}

\author{
Lawrence Yu-Min Liu ${ }^{1,2}$, Zih-Yin Lai ${ }^{1}$, Min-Hsuan $\operatorname{Lin}^{1}$, Yu Shih ${ }^{3}$ and \\ Yung-Jen Chuang ${ }^{1}$ \\ ${ }^{1}$ Department of Medical Science \& Institute of Bioinformatics and Structural Biology, National \\ Tsing Hua University, Hsinchu, 30013, Taiwan \\ ${ }^{2}$ Division of Cardiology, Department of Internal Medicine, Hsinchu Mackay Memorial Hospital, \\ Hsinchu, 30071, Taiwan \\ ${ }^{3}$ Interdisciplinary Program of Life Science, National Tsing Hua University, Hsinchu, 30013, \\ Taiwan \\ drlawrenceliu@gmail.com, laiziyin@gmail.com, \\ daisisky@yahoo.com.tw, samshi77@gmail.com, \\ yjchuang@life.nthu.edu.tw
}

\begin{abstract}
Unlike mammals, adult zebrafish hearts retain a remarkable capacity to regenerate after injury. Since regeneration shares many common molecular pathways with embryonic development, we investigated myocardial remodeling genes and pathways by performing a comparative transcriptomic analysis of zebrafish heart regeneration using a set of known human hereditary heart disease genes related to myocardial hypertrophy during development. We cross-matched human hypertrophic cardiomyopathy-associated genes with a time-course microarray dataset of adult zebrafish heart regeneration. Genes in the expression profiles that were highly elevated in the early phases of myocardial repair and remodeling after injury in zebrafish were identified. These genes were further analyzed with web-based bioinformatics tools to construct a regulatory network revealing potential transcription factors and their upstream receptors. In silico functional analysis of these genes showed that they are involved in cardiomyocyte proliferation and differentiation, angiogenesis, and inflammation-related pathways. The regulatory network indicated that $\beta-2$ microglobulin-mediated signaling may play an important role in myocardial remodeling after injury. This novel cross-species bioinformatics approach to uncover key modulators of zebrafish heart regeneration through human hereditary disease genomic
\end{abstract}


analysis could greatly facilitate the understanding of the evolutionarily conserved cardiac remodeling process.

\section{Introduction}

Myocardial infarction (MI) continues to be the leading cause of death from cardiovascular diseases worldwide. Patients who survive an MI have significantly elevated risks of recurrent MIs, heart failure and death[1]. After injury, the human heart is unable to regenerate itself, and the damaged myocardial tissues are replaced by fibrous scar tissue. Loss of myocardial cells results in decreased contractile function and unfavorable ventricular remodeling, which eventually leads to congestive heart failure and poor clinical outcomes[2],[3]. Additional research to develop new strategies to facilitate cardiomyocyte replacement and functional recovery is urgently needed. However, the current understanding of heart regeneration remains limited.

Unlike that of mammals, the zebrafish heart has an astonishing ability to regenerate itself with minimal scarring after $20 \%$ ventricular resection[4], and the zebrafish has thus become a popular model organism for vertebrate regeneration research. Evidence suggests that this robust regenerative response is mediated by complex developmental pathways, including the TGF- $\beta$, PDGF and FGF signaling pathways[5]. Studies have also indicated that preexisting cardiomyocytes surrounding the injured area undergo dedifferentiation and subsequent proliferation to replace the initial fibrotic scar tissue and eventually restore cardiac function[6, 7]. Surprisingly, neonatal mouse hearts also exhibit such regenerative capabilities through the proliferation of new cardiomyocytes from preexisting cardiomyocytes [8], a process similar to that in adult zebrafish. These observations imply that zebrafish heart regeneration shares common or conserved signaling pathways with mammalian heart development. However, many pathways involved in the complex process of heart regeneration have yet to be elucidated.

To examine the molecular interactions that occur in the myocardial remodeling process during heart regeneration, we developed a novel human hereditary disease gene-based approach to identify significant cardiac regeneration genes using time-course microarray data obtained during zebrafish heart regeneration. A recent study supports zebrafish as a relevant model for studying cardiomyopathy [9]. This approach was based on the hypothesis that during regeneration, injured tissue may undergo a process called embryonic recall[10]. In other words, the damaged heart must undergo cell division and differentiation/dedifferentiation, processes that are likely to be regulated by genes and networks related to critical developmental pathways. Moreover, there is evidence suggesting overlap between the pathways regulating cell division and those regulating cardiac hypertrophy[11]. On the basis of such correlations, causal genes for human hypertrophic cardiomyopathy (HCM), which cause abnormal myocardial cell growth during the developmental process, were exploited to search for myocardial remodeling genes involved in the regeneration process in zebrafish hearts[12, 13]. With these genes, we then systematically analyzed temporal expression profiles to identify key modulators and potential driver pathways. Our study establishes a new bioinformatics approach that could facilitate and guide future studies of heart regeneration given the conservation among developmental and regenerative processes. 


\section{Methods}

\subsection{Gene expression microarray dataset for zebrafish heart regeneration}

Time-course microarray data following wound healing in the zebrafish heart have been published previously (NCBI GEO Dataset: GSE72348)[14]. Briefly, gene expression data for adult zebrafish hearts subjected to ventricular resection were obtained for $0,0.25,1,3,6,10,15,21,28$ dpa (days post amputation). Each time point comprised two biological replicates.

\subsection{Human hereditary disease databases and human-zebrafish homologous gene analysis}

HCM genes were obtained from the HUGO Gene Nomenclature Committee (HGNC) (www.genenames.org), GeneCards (www.genecards.org) and the Genetic Association Database (GAD; geneticassociationdb.nih.gov). These databases provide detailed descriptions of the relationship between HCM and various genes along with the relevant publications.

Homologous gene analysis was performed based on public data from the NCBI (www.ncbi.nlm.nih.gov) website. We mapped human and zebrafish genes that were conserved in both species using online tools from the UniGene (www.ncbi.nlm.nih.gov/unigene), GeneCards, and HomoloGene (www.ncbi.nlm.nih.gov/homologene) databases. A Basic Local Alignment Search Tool (BLAST) analysis was performed for those genes without hits in the databases. All identified zebrafish homologues of HCM-associated genes were included in the analysis of gene expression profile.

\subsection{Microarray data analysis with pattern-matching programs}

To identify specific genes involved in the myocardial remodeling process during heart regeneration, we examined expression profiles related to certain phases of tissue regeneration that are shared by cardiac hypertrophy, which occurs after cardiac injury in zebrafish. Previous studies have already identified the characteristic gene expression profiles of fibroplasia and granulation tissue formation during the wound healing process $[15,16]$. Therefore, we selected two of the specific profiles: the early-phase and acute response profiles. The early-phase profile matches to the expression pattern of fibroplasias and granulation tissue formation, and the acute response profile contains the regulatory genes switching on the genes expression of the early-phase response profile.

Genes involved in the early phase or acute response stage of the heart regeneration process were identified using the Short Time-series Expression Miner (STEM) program (www.cs.cmu.edu/ jernst/stem). Briefly, STEM clustered the genes according to their temporal gene expression patterns in the time-course microarray data into one single profile with the closest pattern[17]. Molecular pathways that were overrepresented in the profiles of genes upregulated after ventricular amputation were identified and analyzed with the PANTHER (Protein Analysis THrough Evolutionary Relationships) system, which classifies proteins based on their molecular functions, biological processes and signaling pathways (www.pantherdb.org). Subsequently, the transcription factors (TFs), TF binding sites (TFBSs) of myocardial remodeling genes, and upstream TF receptors were identified with oPOSSUM (opossum.cisreg.ca/oPOSSUM3) and QIAGEN GeneGlobe (www.qiagen.com/geneglobe). A summary flowchart of the informatics analyses in this study is shown in Figure 1A. 


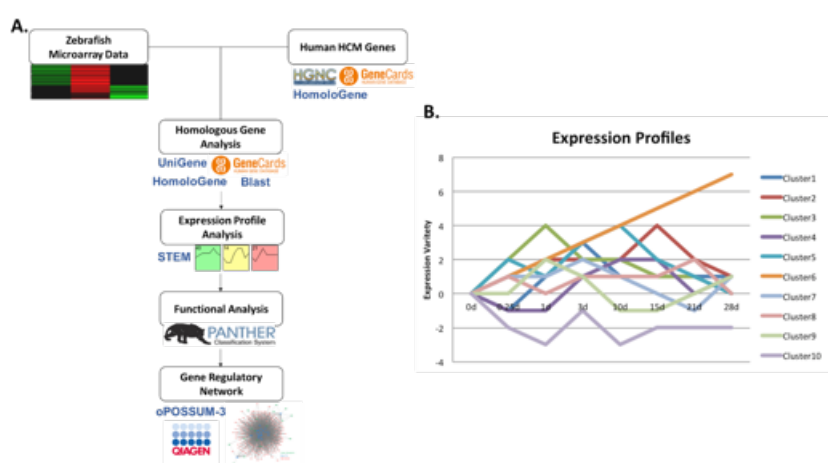

Figure 1: Expression profiles of human hypertrophic cardiomyopathy (HCM) genes that are reactivated during heart remodeling. (A) The expression profiles were obtained by database mining and array matching. The human HCM genes, 337 in total were mined from the GeneCards and GAD databases. By combining the DNA microarray data with zebrafish homologues of HCM genes, which were mapped via NCBI BLAST, we were able to obtain rough expression profiles related to heart remodeling. (B) A simplified diagram of the HCM expression profiles. Analogous expression profiles are classified as a cluster, and here, the profiles can be roughly divided into ten clusters arranged by profile numbers.

\section{Results}

\subsection{Expression profiles of human hypertrophic cardiomyopathy genes reactivated during heart regeneration}

To define the transcriptome of zebrafish heart regeneration and to identify key genes and pathways active in primary processes such as cardiomyocyte differentiation, we screened the zebrafish heart regeneration microarray dataset with human HCM genes. Previous studies have noted that several driver pathways involved in the zebrafish heart development process are reactivated during heart regeneration after injury $[18,19]$. Therefore, $\mathrm{HCM}$, a hereditary disease that is caused by abnormal myocardial development, was exploited to discover transcripts that may not have been otherwise recognized. Accordingly, we mined human HCM genes from the online databases HGNC, GeneCards and GAD using hypertrophic cardiomyopathy as keywords, and obtained a total of 337 HCMassociated genes that were extracted from the relevant research.

All zebrafish homologues of the human HCM-associated genes were mapped with either online HomoloGene and zfin database or the NCBI BLAST analysis. By cross-matching zebrafish homologues of HCM-associated genes with the zebrafish heart regeneration microarray dataset, we obtained complete gene expression profiles arranged in various clusters of coexpression. The genes in these clusters represented candidate zebrafish homologues for human HCM genes that might be reactivated during heart regeneration (Fig. 1B).

\subsection{Identification of the myocardial remodeling genes involved in heart regeneration}

The temporal patterns of profile numbers 40,14 , and 45 corresponded with the early-phase response profile which showed elevated expression at $1 \mathrm{dpa}$, continued to be expressed during the 
remodeling process, and then showed decreased expression at approximately 20-28 dpa. These profiles yielded 48 genes among the matched expression clusters with positive correlations (Table 1; Fig. 2A). Functional analysis showed that, consistent with published findings these genes were mainly enriched in pathways such as the TGF- $\beta$, EGFR, gonadotropin releasing hormone receptor (GnHR), integrin signaling, and inflammation-related pathways (Table 2). Notably, the TGF- $\beta$ signaling pathway has been verified to play an essential role in cell proliferation and differentiation during the zebrafish regeneration process $[7,20]$.

A

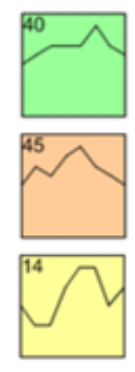

B

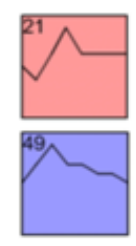

Figure 2: Identification of the myocardial remodeling genes involved in heart regeneration.

(A) Profiles matched by STEM that correspond to the early-phase response profile. There is a total of 48 cardiomyocyte remodeling genes involved in heart regeneration from profiles 40,14 and 45 . $* P<0.01$. (B) Profiles matched by STEM that correspond to the acute response profile that regulate the genes in Figure 2A. There is a total of 105 regulatory gene candidates from profiles 21 and 49 . $* P<0.01$.

(A) Myocardial remodeling genes (corresponding to Fig. 2A)

\begin{tabular}{ll}
\hline ACTA1 & MYLK2 \\
CACNA1C & MYOM1 \\
CACNA2D2 & NDUFAF1 \\
\hline CACNG7 & NDUFS4 \\
CASQ2 & NDUFV2 \\
CHKA & NRAP \\
COX5A & PDHA1 \\
\hline CS & PLN \\
\hline CSRP1 & PRKAG2 \\
\hline EGFR & PVALB \\
FAM188A & SGCB \\
\hline FKBP1B & SLC17A5 \\
FLT1 & SLC25A3 \\
\hline HRAS & TNNC1 \\
\hline HSPB8 & TNNI1 \\
\hline IGFBP3 & TNNT2 \\
\hline IRX4 & TPM1 \\
\hline ITGA5 & TPM4 \\
\hline ITGA8 & \\
\hline MAP1LC3A & \\
\hline MAPK10 & \\
\hline MAPK14 & \\
\hline MEF2A & \\
\hline MEF2C 2 & \\
\hline MSTN & \\
\hline
\end{tabular}

(B) Regulatory genes

\begin{tabular}{llll}
\multicolumn{4}{c}{ corresponding to Fig. 2B) } \\
\hline ACTB & FASLG & MTO1 & STAT3 \\
\hline ACTN1 & FHOD3 & MYL7 & SURF1 \\
ACTN2 & FKTN & MYL9 & TAZ \\
AGK & FXN & MYO6 & TCP1 \\
AGL & GATA4 & NKX2-5 & TGFB3 \\
\hline ANXA5 & GLA & NOS2 & TGM2 \\
APEH & GNAI1 & NPPA & TIMP2 \\
AR & GRB2 & NR3C2 & TLN1 \\
AUH & HTR2A & PCNA & TNC \\
B3GAT3 & IFNA2 & PDLIM3 & TP53 \\
BIRC5 & ILK & PMM2 & TP63 \\
\hline CACNA2D1 & INS & POMC & TPM3 \\
CACNB4 & ITGA3 & PRKAB1 & TTN \\
\hline CACNG2 & ITGAV & PRKAB2 & VCL \\
CACNG6 & ITGB1 & PRKACA & VDAC1 \\
\hline CAMK2G & ITGB5 & PRKAG1 & \\
\hline CAV1 & JAK2 & PTEN & \\
\hline CAV3 & JUN & PTPN11 & \\
\hline CCL2 & KRAS & PYGB & \\
\hline CD36 & LAMP2 & REN & \\
\hline CIB1 & LARP7 & RYR1 & \\
\hline COL1A1 & LMNA & SCO2 & \\
\hline COL1A2 & MAP2K1 & SGCD & \\
\hline COX15 & MAP2K2 & SGCG & \\
\hline CTNNB1 & MAPK7 & SLC22A5 & \\
\hline DAG1 & MAPK8 & SLC6A4 & \\
\hline DES & MCM10 & SLC9A1 & \\
\hline
\end{tabular}




\begin{tabular}{|c|c|c|c|}
\hline MYBPC3 & DMD & MMP2 & SMC1A \\
\hline MYH6 & ERBB2 & MRPL3 & SOS1 \\
\hline MYH7 & ESR1 & MRPL44 & SRI \\
\hline
\end{tabular}

Table 1: The myocardial remodeling genes identified by STEM.

\begin{tabular}{ll}
\hline Pathway & Genes \\
\hline TGF- $\beta$ & HRAS, MAPK10, MAPK14, MSTN \\
EGFR & HRAS, MAPK10, MAPK14, MSTN \\
GnRH & CACNA1C, EGFR, HRAS, MAPK14, PRKAG2 \\
Integrin & HRAS, ITGA5, ITGA8, MAPK10 \\
Cytoskeletal regulation & ACTA1, MYH6, MYH7, MYLK2 \\
Inflammation & ACTA1, MYH7, MYHR, MYLK2 \\
\hline nAChRs & ACTA1, CACNA1C, MYH6, MYH7 \\
\hline B & \\
\hline Pathway & Genes \\
\hline Angiogenesis & BIRC5, CTNNB1, JUN, KRAS, MAP2K1, MAP2K2, MAPK8, PTPN11, SOS1, \\
CCKR & STAT3 \\
GnRH & CTNNB1, ITGAV, ITGB1, JAK2, JUN, MAP2K1, MAP2K2, MAPK7, MAPK8, \\
& PRKACA, PTEN, PTPN11, RYR1, SOS1, STAT3 \\
Inflammation & ANXA5, AR, CAV1, CTNNB1, GATA4, GNAI1, ITGB1, JUN, MAP2K1, \\
& MAP2K2, MAPK8, PRKAB1, PRKAB2, PRKAG1, SOS1, STAT3, VCL \\
\hline Integrin & ACTB, CAMK2G, CCL2, GNAI1, ITGB1, JAK2, JUN, KRAS, PRKACA, \\
\hline PDGF & PTEN, SOS1, STAT3, \\
\hline
\end{tabular}

Table 2: Representative myocardial remodeling genes involved in heart regeneration and their related pathways from PANTHER. (A) Analysis of the early-phase response genes from PANTHER. The first column includes the related pathways, and the second column includes the genes participating in each pathway. (B) Analysis of the acute response genes from PANTHER.

The acute response profile contained regulatory genes that trigger the expression of genes in the early-phase profile. A total of 109 genes identified in the acute response stage from STEM profile numbers 21 and 49 (Table 1; Fig. 2B) were involved in the PDGF and angiogenesis-related pathways as well as in the $\mathrm{GnRH}$, integrin signaling and inflammation-related pathways identified for the earlyphase profile (Table 2).

\subsection{Construction of the gene regulatory network for myocardial recovery}

To further investigate the myocardial remodeling process in the early phase and the acute response stage of regeneration, we constructed a regulatory network using the TF binding site-detecting tool oPOSSUM-3. Unlike other TF-detecting tools, oPOSSUM analyzes whole sets of genes to identify the TFs of coexpressed genes. Moreover, the TFs are scored based on a comparison between the number of detected TFBS nucleotides in the target genes and the oPOSSUM background dataset to provide the significance of the TF results. Accordingly, we obtained 116 TFs that were potential regulatory TFs of our previously selected genes in the early-phase and acute response profiles and ranked them by Z-score (Table 3). A higher Z-score indicates an increased rate of occurrence of a given TFBS in the foreground sequence set of co-expressed genes using a simple binominal 
distribution model[21, 22]. Again, the transcriptome analysis yielded key molecules involved in both development and heart regeneration, including MEF2A and Nkx2-5[16, 23]. These results indicate that the human HCM disease gene-based search approach can effectively uncover myocardial remodeling-related targets that are important during heart regeneration and thus support the use of our methods to facilitate heart regeneration research.

\begin{tabular}{|l|l|}
\hline \multicolumn{1}{|c|}{ TF } & Z-score \\
\hline MEF2A & 26.217 \\
\hline TBP & 26.18 \\
\hline Nkx2-5 & 23.37 \\
\hline SRF & 21.782 \\
\hline FOXI1 & 21.658 \\
\hline Pdx1 & 21.17 \\
\hline HOXA5 & 18.841 \\
\hline Nobox & 18.697 \\
\hline Gata1 & 18.503 \\
\hline SRY & 17.239 \\
\hline Prrx2 & 16.632 \\
\hline FOXA1 & 16.631 \\
\hline NKX3-1 & 16.148 \\
\hline Nkx3-2 & 15.967 \\
\hline Sox5 & 15.465 \\
\hline ARID3A & 15.381 \\
\hline Foxa2 & 15.204 \\
\hline FOXO3 & 14.329 \\
\hline AP1 & 13.836 \\
\hline Pou5f1 & 13.793 \\
\hline Sox17 & 12.885 \\
\hline SPIB & 12.661 \\
\hline Foxd3 & 12.404 \\
\hline ELF5 & 11.948 \\
\hline SOX9 & 11.732 \\
\hline PPARG::R & 11.716 \\
XRA & 11.153 \\
\hline RUNX1 & 10.654 \\
\hline Foxq1 & 10.599 \\
\hline FOXF2 & 10.565 \\
\hline HNF1A & \\
\hline & \\
\hline
\end{tabular}

\begin{tabular}{|l|c|}
\hline \multicolumn{1}{|c|}{ TF } & Z-score \\
\hline RORA_2 & 10.544 \\
\hline RORA_1 & 10.529 \\
\hline CEBPA & 10.524 \\
\hline PBX1 & 10.176 \\
\hline ESR1 & 10.038 \\
\hline $\begin{array}{l}\text { Tal1::Gata } \\
\text { 1 }\end{array}$ & 9.912 \\
\hline NFE2L2 & 9.355 \\
\hline Lhx3 & 9.105 \\
\hline FEV & 9.062 \\
\hline Pax6 & 8.642 \\
\hline FOXD1 & 8.58 \\
\hline SPI1 & 8.204 \\
\hline $\begin{array}{l}\text { Hand1::Tcfe } \\
\text { 2a }\end{array}$ & 8.063 \\
\hline NR4A2 & 7.624 \\
\hline HNF1B & 7.57 \\
\hline NR2F1 & 7.481 \\
\hline Spz1 & 7.383 \\
\hline PPARG & 7.214 \\
\hline $\begin{array}{l}\text { NF- } \\
\text { kappaB }\end{array}$ & 6.977 \\
\hline MIZF & 6.915 \\
\hline Arnt::Ahr & 6.696 \\
\hline CTCF & 6.641 \\
\hline NFIL3 & 6.28 \\
\hline NFATC2 & 6.247 \\
\hline TEAD1 & 6.146 \\
\hline RREB1 & 6.099 \\
\hline ESR2 & 5.932 \\
\hline EBF1 & 5.664 \\
\hline Esrrb & 5.551 \\
\hline $\begin{array}{l}\text { Ddit3::Ceb } \\
\text { pa }\end{array}$ & 5.536 \\
\hline fators \\
\hline
\end{tabular}

\begin{tabular}{|l|r|}
\hline \multicolumn{1}{|c|}{ TF } & Z-score \\
\hline SP1 & 5.369 \\
\hline Gfi & 5.334 \\
\hline REL & 5.162 \\
\hline MZF1_5-13 & 4.903 \\
\hline STAT1 & 4.822 \\
\hline NR3C1 & 4.65 \\
\hline RELA & 4.603 \\
\hline HNF4A & 4.575 \\
\hline Sox2 & 3.805 \\
\hline YY1 & 3.547 \\
\hline IRF2 & 3.325 \\
\hline Nr2e3 & 3.239 \\
\hline znf143 & 3.132 \\
\hline HLF & 3.008 \\
\hline T & 2.894 \\
\hline CREB1 & 2.772 \\
\hline Myf & 2.723 \\
\hline Zfp423 & 2.632 \\
\hline Myb & 2.494 \\
\hline NFKB1 & 2.461 \\
\hline EWSR1- & 2.336 \\
\hline FLI1 & 2.157 \\
\hline IRF1 & 2.092 \\
\hline Klf4 & 2.045 \\
\hline INSM1 & 1.422 \\
\hline TAL1::TCF3 & 1.015 \\
\hline TLX1::NFIC & 0.495 \\
\hline MZF1_1-4 & 0.48 \\
\hline Ar & \\
\hline Evi1 & \\
\hline PLAG1 & \\
\hline
\end{tabular}

\begin{tabular}{|l|c|}
\hline \multicolumn{1}{|c|}{ TF } & Z-score \\
\hline $\begin{array}{l}\text { RXRA::VD } \\
\text { R }\end{array}$ & 0.09 \\
\hline ZNF354C & -0.089 \\
\hline ZEB1 & -0.111 \\
\hline ELK1 & -0.476 \\
\hline E2F1 & -0.522 \\
\hline RXR::RAR_ & -0.566 \\
DR5 & -0.942 \\
\hline Pax4 & -1.103 \\
\hline NHLH1 & -1.529 \\
\hline REST & -1.675 \\
\hline TP53 & -1.882 \\
\hline Tcfcp211 & -1.971 \\
\hline Stat3 & -2.119 \\
\hline MAX & -3.243 \\
\hline Zfx & -3.378 \\
\hline MYC::MA & -3.598 \\
X & -3.804 \\
\hline Egr1 & -3.852 \\
\hline USF1 & -3.886 \\
\hline HIF1A::AR \\
NT & -4.08 \\
\hline ELK4 & -4.313 \\
\hline Arnt & -5.702 \\
\hline NR1H2::R \\
XRA & -7.311 \\
\hline NFYA & -7.369 \\
\hline Pax5 & -8.078 \\
\hline GABPA & \\
\hline Myc & \\
\hline Mycn & \\
\hline & \\
\hline
\end{tabular}

Table 3: Transcription factors identified by oPOSSUM.

\section{$3.4 \quad \beta$-2-Microglobulin signaling pathways may play a significant role in regulating myocardial remodeling during heart regeneration}

We next identified the upstream receptor signaling pathways that lead to the activation of these TFs using QIAGEN GeneGlobe. The QIAGEN GeneGlobe database provides interaction networks for target gene inputs that include upstream or downstream proteins and chemicals, and these results 
can be further filtered by directionality and molecular type. Through this analysis, several upstream receptors of our TFs were revealed and were ranked by the TF Z-scores (Table 4).

\begin{tabular}{|l|c|}
\hline \multicolumn{1}{|c|}{ Receptors } & Z-score \\
\hline B2M & 26.217 \\
\hline FAS & 21.782 \\
\hline IL1R1 & 21.782 \\
\hline $\begin{array}{l}\text { TNFRSF12 } \\
\text { A }\end{array}$ & 21.782 \\
\hline PTCH1 & 21.17 \\
\hline KIT & 18.503 \\
\hline EPOR & 18.503 \\
\hline BTNL2 & 18.503 \\
\hline CAV1 & 16.631 \\
\hline LRP5 & 16.631 \\
\hline
\end{tabular}

\begin{tabular}{|l|r|}
\hline Receptors & Z-score \\
\hline MUC1 & 14.329 \\
\hline CTLA4 & 14.329 \\
\hline IL6ST & 14.329 \\
\hline IGF1R & 14.329 \\
\hline $\begin{array}{l}\text { TNFRSF1 } \\
\text { 1A }\end{array}$ & 13.793 \\
\hline Pdgfr & 13.793 \\
\hline Notch & 13.793 \\
\hline GPC1 & 12.885 \\
\hline CD247 & 11.948 \\
\hline SFRP1 & 11.732 \\
\hline
\end{tabular}

\begin{tabular}{|l|r|}
\hline \multicolumn{1}{|c|}{ Receptors } & \multicolumn{1}{c|}{ Z-score } \\
\hline ITGB3 & 11.153 \\
\hline CD28 & 10.544 \\
\hline TLR9 & 10.524 \\
\hline PRLR & 10.038 \\
\hline LGR4 & 10.038 \\
\hline TNFRSF1A & 9.355 \\
\hline Fgfr & 8.642 \\
\hline TLR4 & 8.204 \\
\hline TREM1 & 7.624 \\
\hline NCR2 & 7.624 \\
\hline
\end{tabular}

\begin{tabular}{|l|r|}
\hline \multicolumn{1}{|c|}{ Receptors } & Z-score \\
\hline CD36 & 7.214 \\
\hline LEPR & 7.214 \\
\hline ICAM3 & 7.214 \\
\hline KLRK1 & 7.214 \\
\hline BAFFR & 6.977 \\
\hline BCR & 6.977 \\
\hline TCR & 6.977 \\
\hline TLRs & 6.977 \\
\hline IL-1R & 6.977 \\
\hline GFR & 6.977 \\
\hline
\end{tabular}

Table 4: Upstream receptors that lead to the activation of target TFs.

Our data showed that a $\beta$-2-microglobulin (B2M)-related signaling pathway was likely to be a potential modulator of zebrafish myocardial remodeling after ventricular resection. B2M has been found to mediate cell apoptosis and survival by regulating the expression of hormone and growth factors[24]. A gene regulatory network for myocardial recovery was constructed (Fig. 3) including these genes, TFs and the associated upstream receptor proteins identified by QIAGEN GeneGlobe. Only the top 20 ranked TFs with upstream receptors are shown.

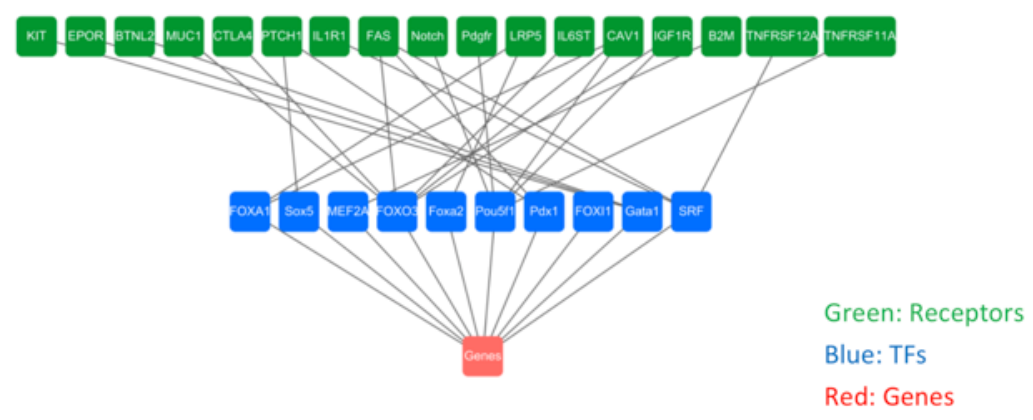

Figure 3: The gene regulatory network of myocardial recovery.

\section{Discussion}

In this study, we used a human developmental hereditary disease (i.e., hypertrophic cardiomyopathy) gene set and a zebrafish heart regeneration microarray dataset to investigate the myocardial remodeling process during heart regeneration. HCM genes were crossed-matched with zebrafish heart regeneration transcriptome profiles, and HCM expression profiles were used to search for myocardial remodeling modulators. The TFs and upstream receptors of the identified myocardial remodeling genes were further analyzed with bioinformatics databases and tools to enable the identification of the modulators.

The same zebrafish microarray dataset has previously been used to reveal organ-specific regenerative strategies[14] that include the heart-specific pathways also observed in our acute and early-phase response profiles derived from the selected myocardial remodeling genes. In this study, 
gene regulatory network analysis further revealed that B2M signaling pathways may play an important role in myocardial remodeling during heart regeneration (Fig. 4).

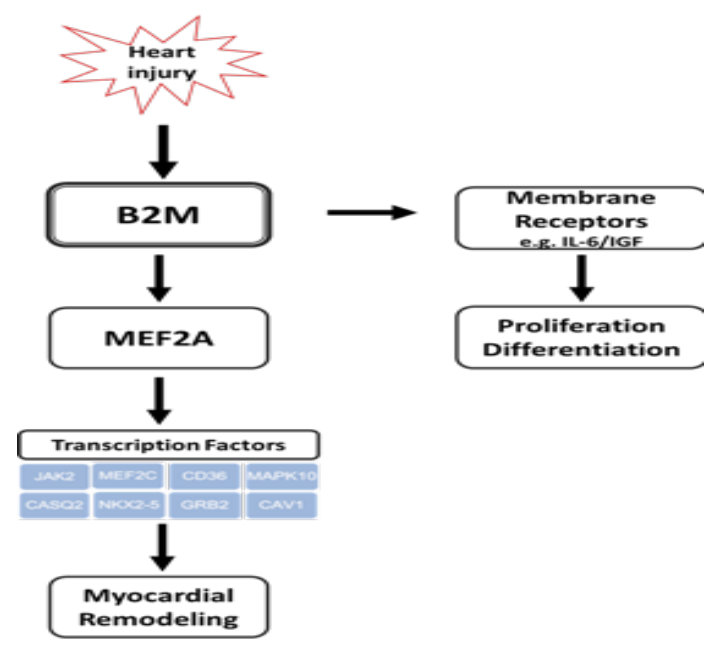

Figure 4: Proposed gene regulatory network of B2M myocardial remodeling during regeneration. After myocardial injury, related signals bind to $\mathrm{B} 2 \mathrm{M}$, promoting a cascade of interacting kinase proteins or molecules that activate other membrane receptors or TFs, such as MEF2A, and then further regulate downstream genes to remodel the injured myocardium.

B2M composes the light chains of major histocompatibility complex class I (MHC1) molecules and is an active part of the immune system[25]. MHC1 has been reported to regulate neuronal synapses during development via the MHC1-MEF2 signaling pathway, as verified by the reduction in MEF2 activity in B2M RNAi knockdown mice[26]. MEF2 is an essential cardiac TF that promotes the generation of cardiomyocytes from cardiac or skin fibroblasts[27, 28]. MEF2 homologues in zebrafish are also essential in cardiomyocyte differentiation, and their role is believed to be conserved throughout vertebrate hearts[29]. Moreover, B2M activates the epithelial-tomesenchymal transition (EMT) and promotes the growth and migration of mesenchymal stem cells in cancer $[30,31]$. Thus, although the mechanism by which $\mathrm{B} 2 \mathrm{M}$ mediates myocardial remodeling is not fully understood, the potentially novel functional role of $\mathrm{B} 2 \mathrm{M}$ in heart regeneration is worth examining in future studies.

Multiple molecules identified by our approach have already been shown to be important in heart repair, including erythropoietin (EPO) and c-kit (KIT). EPO is a growth hormone that binds to the transmembrane receptor EPOR, and EPO-responsive cardiomyogenic cells can contribute to heart repair in MI mice[32]. Additionally, c-kit-positive cardiac stem cells have been found to be necessary and sufficient for functional cardiac regeneration and repair[33]. Interestingly, another receptor at the top of our target list, caveolin 1 (CAV1), was recently demonstrated in a transcriptomic analysis of epicardial lineage cells to be an essential factor in injury-induced cardiomyocyte proliferation and heart regeneration[34]. Similarly, a different transcriptomic analysis study revealed that specific enhancer regulatory elements (e.g. leptin receptor LEPR) can direct the regeneration-induced activation of gene expression in zebrafish and neonatal mouse tissues[35]. These studies provide evidences that our method is sufficient to predict candidate targets with regulatory roles in heart regeneration.

This study described a new systematic approach to identify conserved modulators of heart regeneration based on findings from developmental biology. The zebrafish has become a new model 
to study the genetic basis of cardiomyopathy because of availability of both embryonic and adult models and well conserved diploid genome among vertebrates[36]. This human hereditary disease gene-based method enables the construction of gene regulatory networks and the identification of growth factors that play important roles during heart regeneration. This type of analysis can be expanded to different cardiovascular diseases to increase our understanding of complex molecular processes and to prioritize homologues for each disease-associated gene. For example, it would be worthwhile to apply familial arrhythmia-associated genes to explore potential pathways involved in the synchronization of cardiac contraction. However, this approach has several limitations. Since the interactions in the regulatory network are obtained mainly from online databases, empirical experiments are still necessary to validate the findings. Moreover, the selection of different processes and time frames during regeneration may bypass the unique characteristics and pathogeneses of human diseases. In addition, the use of RNA extracted from the whole heart makes it difficult to identify cell or tissue-specific gene regulation that occurs during the process.

Currently, heart regeneration is studied through three main approaches[37]. The first mainstream approach focuses on stem cells and attempts to target cells or tissue grafts to the injured area. The second approach intends to reprogram scar tissue into new functional myocardium through the use of TFs or miRNA. The final approach focuses on stimulating existing cardiomyocytes or resident cardiac cells with growth factors or small molecules to initiate heart regeneration. In investigating heart regeneration, our human disease gene-based method can be applied to complement the second and third approaches. The TFs or miRNA optimal for scar reprogramming could be identified by analyzing scar tissue formation-related disease genes and a heart regeneration microarray dataset, while growth factors or mediator molecules could be identified based on a gene regulatory network constructed from relevant cardiovascular diseases. With the rapid advancement of bioinformatics databases and tools, this human disease gene-based method can thus potentially be very useful in studies on the mechanisms of heart regeneration and in the field of regenerative cardiac medicine.

In conclusion, our results encourage follow-up studies using human disease gene-based analysis to identify key modulators involved in post-MI recovery. Applying our search approach to genes involved in different diseases may enable more effective investigation and may facilitate the understanding of the specific process involved in heart regeneration.

\section{References}

[1] M. Writing Group et al., "Heart Disease and Stroke Statistics-2016 Update: A Report From the American Heart Association," Circulation, vol. 133, no. 4, pp. e38-360, Jan 262016.

[2] M. A. Pfeffer and E. Braunwald, "Ventricular remodeling after myocardial infarction. Experimental observations and clinical implications," Circulation, vol. 81, no. 4, pp. 116172, Apr 1990.

[3] E. Tzahor and K. D. Poss, "Cardiac regeneration strategies: Staying young at heart," Science, vol. 356, no. 6342, pp. 1035-1039, Jun 92017.

[4] K. D. Poss, L. G. Wilson, and M. T. Keating, "Heart regeneration in zebrafish," Science, vol. 298, no. 5601, pp. 2188-90, Dec 132002.

[5] J. Cao and K. D. Poss, "The epicardium as a hub for heart regeneration," Nat Rev Cardiol, Jun 272018.

[6] C. Jopling, E. Sleep, M. Raya, M. Marti, A. Raya, and J. C. Izpisua Belmonte, "Zebrafish heart regeneration occurs by cardiomyocyte dedifferentiation and proliferation," Nature, vol. 464, no. 7288, pp. 606-9, Mar 252010. 
[7] F. Chablais and A. Jazwinska, "The regenerative capacity of the zebrafish heart is dependent on TGFbeta signaling," Development, vol. 139, no. 11, pp. 1921-30, Jun 2012.

[8] E. R. Porrello et al., "Transient regenerative potential of the neonatal mouse heart," Science, vol. 331, no. 6020, pp. 1078-80, Feb 252011.

[9] Y. H. Shih et al., "Cardiac transcriptome and dilated cardiomyopathy genes in zebrafish," Circ Cardiovasc Genet, vol. 8, no. 2, pp. 261-9, Apr 2015.

[10] W. Mohl, D. Milasinovic, T. Aschacher, A. Jusic, A. Maimaitiaili, and F. Rattay, "The Hypothesis of "Embryonic Recall": Mechanotransduction as Common Denominator Linking Normal Cardiogenesis to Recovery in Adult Failing Hearts," Journal of Cardiovascular Development and Disease, vol. 1, no. 1, pp. 73-82, 2014.

[11] P. Ahuja, P. Sdek, and W. R. MacLellan, "Cardiac myocyte cell cycle control in development, disease, and regeneration," Physiol Rev, vol. 87, no. 2, pp. 521-44, Apr 2007.

[12] H. Sisakian, "Cardiomyopathies: Evolution of pathogenesis concepts and potential for new therapies," World J Cardiol, vol. 6, no. 6, pp. 478-94, Jun 262014.

[13] M. W. Chung, T. Tsoutsman, and C. Semsarian, "Hypertrophic cardiomyopathy: from gene defect to clinical disease," Cell Res, vol. 13, no. 1, pp. 9-20, Feb 2003.

[14] F. Y. Liu et al., "Uncovering the regeneration strategies of zebrafish organs: a comprehensive systems biology study on heart, cerebellum, fin, and retina regeneration," BMC Syst Biol, vol. 12, no. Suppl 2, p. 29, Mar 192018.

[15] J. T. Shin and M. C. Fishman, "From Zebrafish to human: modular medical models," Annu Rev Genomics Hum Genet, vol. 3, pp. 311-40, 2002.

[16] A. Lepilina et al., "A dynamic epicardial injury response supports progenitor cell activity during zebrafish heart regeneration," Cell, vol. 127, no. 3, pp. 607-19, Nov 32006.

[17] J. Ernst and Z. Bar-Joseph, "STEM: a tool for the analysis of short time series gene expression data," BMC Bioinformatics, vol. 7, p. 191, Apr 52006.

[18] W. Y. Choi et al., "In vivo monitoring of cardiomyocyte proliferation to identify chemical modifiers of heart regeneration," Development, vol. 140, no. 3, pp. 660-6, Feb 12013.

[19] V. Gupta, M. Gemberling, R. Karra, G. E. Rosenfeld, T. Evans, and K. D. Poss, "An injuryresponsive gata4 program shapes the zebrafish cardiac ventricle," Curr Biol, vol. 23, no. 13, pp. 1221-7, Jul 82013.

[20] D. Dogra, S. Ahuja, H. T. Kim, S. J. Rasouli, D. Y. R. Stainier, and S. Reischauer, "Opposite effects of Activin type 2 receptor ligands on cardiomyocyte proliferation during development and repair," Nat Commun, vol. 8, no. 1, p. 1902, Dec 12017.

[21] S. J. Ho Sui et al., "oPOSSUM: identification of over-represented transcription factor binding sites in co-expressed genes," Nucleic Acids Res, vol. 33, no. 10, pp. 3154-64, 2005.

[22] A. T. Kwon, D. J. Arenillas, R. Worsley Hunt, and W. W. Wasserman, "oPOSSUM-3: advanced analysis of regulatory motif over-representation across genes or ChIP-Seq datasets," G3 (Bethesda), vol. 2, no. 9, pp. 987-1002, Sep 2012.

[23] J. W. Vincentz, R. M. Barnes, B. A. Firulli, S. J. Conway, and A. B. Firulli, "Cooperative interaction of Nkx2.5 and Mef2c transcription factors during heart development," Dev Dyn, vol. 237, no. 12, pp. 3809-19, Dec 2008.

[24] J. Yang et al., "Anti beta2-microglobulin monoclonal antibodies induce apoptosis in myeloma cells by recruiting MHC class I to and excluding growth and survival cytokine receptors from lipid rafts," Blood, vol. 110, no. 8, pp. 3028-35, Oct 152007.

[25] M. Zijlstra, M. Bix, N. E. Simister, J. M. Loring, D. H. Raulet, and R. Jaenisch, "Beta 2microglobulin deficient mice lack CD4-8+ cytolytic T cells," Nature, vol. 344, no. 6268, pp. 742-6, Apr 191990.

[26] B. M. Elmer, M. L. Estes, S. L. Barrow, and A. K. McAllister, "MHCI requires MEF2 transcription factors to negatively regulate synapse density during development and in disease," J Neurosci, vol. 33, no. 34, pp. 13791-804, Aug 212013. 
[27] M. Ieda et al., "Direct reprogramming of fibroblasts into functional cardiomyocytes by defined factors," Cell, vol. 142, no. 3, pp. 375-86, Aug 62010.

[28] L. Qian et al., "In vivo reprogramming of murine cardiac fibroblasts into induced cardiomyocytes," Nature, vol. 485, no. 7400, pp. 593-8, May 312012.

[29] Y. Hinits, L. Pan, C. Walker, J. Dowd, C. B. Moens, and S. M. Hughes, "Zebrafish Mef2ca and Mef2cb are essential for both first and second heart field cardiomyocyte differentiation," Dev Biol, vol. 369, no. 2, pp. 199-210, Sep 152012.

[30] S. Josson et al., "beta2-microglobulin induces epithelial to mesenchymal transition and confers cancer lethality and bone metastasis in human cancer cells," Cancer Res, vol. 71, no. 7, pp. 2600-10, Apr 12011.

[31] C. Shi, Y. Zhu, Y. Su, L. W. Chung, and T. Cheng, "Beta2-microglobulin: emerging as a promising cancer therapeutic target," Drug Discov Today, vol. 14, no. 1-2, pp. 25-30, Jan 2009.

[32] M. P. Zafiriou et al., "Erythropoietin responsive cardiomyogenic cells contribute to heart repair post myocardial infarction," Stem Cells, vol. 32, no. 9, pp. 2480-91, Sep 2014.

[33] G. M. Ellison et al., "Adult c-kit(pos) cardiac stem cells are necessary and sufficient for functional cardiac regeneration and repair," Cell, vol. 154, no. 4, pp. 827-42, Aug 152013.

[34] J. Cao et al., "Single epicardial cell transcriptome sequencing identifies Caveolin 1 as an essential factor in zebrafish heart regeneration," Development, vol. 143, no. 2, pp. 232-43, Jan 152016.

[35] J. Kang et al., "Modulation of tissue repair by regeneration enhancer elements," Nature, vol. 532, no. 7598, pp. 201-6, Apr 142016.

[36] A. V. Dvornikov, P. P. de Tombe, and X. Xu, "Phenotyping cardiomyopathy in adult zebrafish," Prog Biophys Mol Biol, vol. 138, pp. 116-125, Oct 2018.

[37] S. A. Doppler, M. A. Deutsch, R. Lange, and M. Krane, "Cardiac regeneration: current therapies-future concepts," J Thorac Dis, vol. 5, no. 5, pp. 683-97, Oct 2013. 